\title{
A Method of Designing Nonlinear Observers for Squirrel-cage Induction Generators in Windmill Power Systems
}

\author{
Student Member Youjiang Long \\ (University of the Ryukyus) \\ Non-member Shigeru Hanba \\ (University of the Ryukyus) \\ Member \\ Hayao Miyagi \\ (University of the Ryukyus) \\ Member \\ Katsumi Yamashita \\ (University of the Ryukyus)
}

This paper presents a new method of designing nonlinear observers for squirrel-cage induction generators in the windmill power systems. The control law which globally stabilizes the currents, angular velocity and pitch angle under full state measurement is determined by Lyapunov's direct method. Exponentially stable observers for the induction generator subsystem in the windmill power system, which is modeled as a bilinear system, is proposed. The design of the proposed observer is based on Lyapunov's direct method, and its estimation error decays to zero exponentially irrespective of the input. It is also shown that the observer-based extended system is globally stable.

Keywords: Nonlinear observer, Induction generator, Windmill power system.

\section{Introduction}

Squirrel-cage induction generators are widely used as generators in windmill power systems, because they are inexpensive, have high durability, and are capable of operating asynchronously with power systems. However, induction generators suffer the drawback that they cause transient rush currents that are several times larger than the rated value, and wind energy is influenced by geographic and weather conditions, the effect of the fluctuation of the power supplied by the windmill generator is serious ${ }^{(1)}$. Therefore, in these cases, it is important to regulate the power to a rated value. This motivates the study of transient rush current/voltage attenuation in the windpower generator systems ${ }^{(2)}$.

In order to control windmill power systems, it is necessary to measure the stator currents, the rotor currents, the electric angular velocity of squirrel-cage induction generators, and the pitch angle of the blade of the windmill. However, among them the rotor currents are difficult to measure. To construct a controller which can be easily implemented to a real windmill generator, it is necessary to design an observer which estimates rotor currents from the other state variables which are measurable. As windmill power systems have strong nonlinearity, it is necessary to use a system of nonlinear differential equations as a model of the system ${ }^{(2)}$. Especially, the subsystem which is related with the rotor currents can be modeled as a bilinear system if the electric angular speed is considered as an input, so that we concentrate on the approach of designing an observer for the bilinear system

In the case of that not all of the state variables are available, the use of some state estimators is necessary in order to implement a state feedback control system. Unlike linear systems the observability of bilinear systems is affected by the inputs, so the observer whose estimation error depends on inputs can work satisfactorily only in preassigned conditions. In this paper, the design of the observer is based on the solution of simultaneous Lyapunov equations ${ }^{(3)(4)}$. It is proved that the estimation error decays to zero exponentially irrespective of the input, although the estimation error itself depends on the input.

The paper is organized as follows: Section 2 presents the basic concept of windmill power system, in which the relation between generated power of the windmill and the pitch angle versus wind speed, the voltage equations of the induction generator and the dynamic equations of the windmill are introduced. Section 3 describes state equations of the windmill power system and section 4 presents the design of a controller under full-state measurement, which regulates the windmill power system states at their rated values. The observer for the generator subsystem in the windmill power system is proposed in section 5 . Section 6 considers the stability of the observer-based extended windmill power system. Simulation results are then presented in section 7 .

\section{Windmill power system}

The conceptual figure of a windmill power system is illustrated in Fig.1. The windmill generator, which consists of the propeller windmill, the gearbox, and the control equipment for the pitch angle of the blade, is connected to infinite bus through the transformer and the power-transmission line.

2.1 Control of the pitch angle of the blade in the windmill The basic control movements of the 


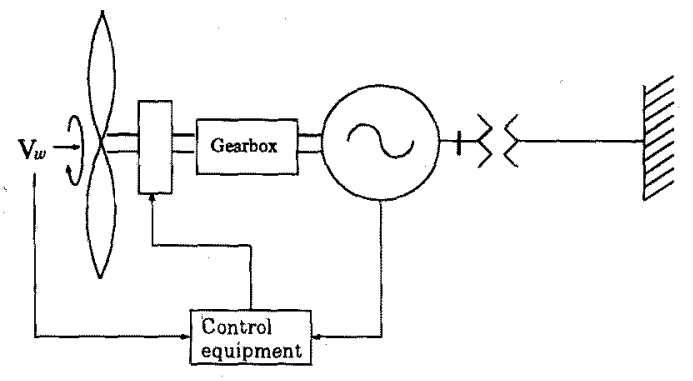

Fig. 1. Windmill generator system.

pitch angle of the blade in the windmill induction generator system are determined by dividing the speed of wind $V_{w}(t)[\mathrm{m} / \mathrm{sec}]$ into the following four ranges:

(i) less than the cut-in speed $V_{i n}(t)$;

(ii) from the cut-in speed to the rated speed $V_{\text {in }}(t) \leq V_{w}(t)<V_{w}^{*}(t)$

(iii) from the rated speed to the cut-out speed $V_{w}^{*}(t) \leq V_{w}(t)<V_{\text {out }}(t)$

(iv) more than the cut-out speed $V_{w}(t) \geq V_{\text {out }}(t)$.

The relation between the pitch angle $\beta$ and the generator power $P_{e}$ of windmill versus wind speed is shown in Fig.2.

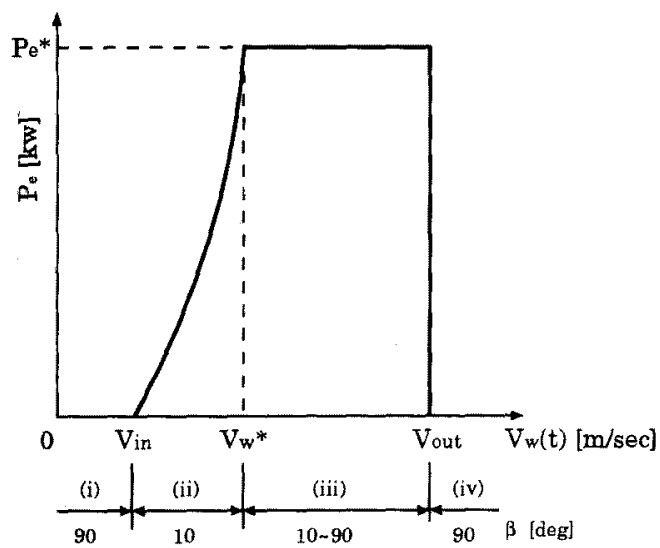

Fig. 2. Relation between generated power of windmill and pitch angle versus wind speed.

(1) When the wind speed is less than the cut-in speed, the pitch angle $\beta$ is forced to 90 [deg] and the windmill is stopped by releasing wind energy.

(2) When the wind speed is in the range from the cut-in speed to the rated speed, if the average wind speed is over the cut-in speed for $10 \mathrm{~min}$ utes, the pitch angle is set to 10 [deg] and the windmill induction generator is connected to the power system. In order to make use of wind energy to the maximum, the pitch angle is fixed at $10[\mathrm{deg}]$ while the wind speed is in this range. As shown in Fig. 2, the output of the induction generator is determined depending on wind speed.

(3) When the wind speed is in the range from the rated speed to the cut-out speed, in order to keep the rated power output and the rated speed of the induction generator within limits, the pitch angle of the blade is controlled by the hydraulic system and it varies from $10[\mathrm{deg}]$ to $90[\mathrm{deg}]$.

(4) When the wind speed exceeds the cut-out speed, as in the case (i), the pitch angle is set to $90[\mathrm{deg}]$ and the windmill is forced to stop by releasing wind energy.

In this paper, we design the controller and observer for case (iii). The pitch angie is controlled by a hydraulic system, its rate is limited to $|d \beta / d t| \leq 10[\mathrm{deg} / \mathrm{sec}]$ due to the limited response speed of the hydraulic system.

2.2 Model of the windmill power system In windmill power systems, the voltage equations of an induction generator are expressed in state variables which are transformed from three-phase to a rotating $(\mathrm{d}-\mathrm{q})$ axis frame with synchronous speed ${ }^{(7)}$. The hydraulic system which adjusts the pitch angle of the blade is modeled by a strictly proper stable first order system. Because the step-up transformer is used between the windmill power system and the power-transmission line, the voltage and impedance in the side of infinite bus is calculated to the side of the generator system, so that, the following dynamic equations of the windmill power system are obtained ${ }^{(2)(3)}$

$v_{s d}=\left(\tilde{R}_{s}+\tilde{L}_{s} \frac{d}{d t}\right) i_{s d}+M \frac{d i_{r d}}{d t}-\omega_{0} \widetilde{L}_{s} i_{s q}-\omega_{0} M i_{r q}$

$v_{s q}=\left(\widetilde{R}_{s}+\widetilde{L}_{s} \frac{d}{d t}\right) i_{s q}+M \frac{d i_{r q}}{d t}+\omega_{0} \tilde{L}_{s} i_{s d}+\omega_{0} M i_{r d}$,

$0=M \frac{d i_{s d}}{d t}-s \omega_{0} M i_{s q}+\left(R_{r}+L_{r} \frac{d}{d t}\right) i_{r d}-s \omega_{0} L_{r} i_{r q}$,

$0=s \omega_{0} M i_{s d}+M \frac{d i_{s q}}{d t}+s w_{0} L_{r} i_{r d}+\left(R_{r}+L_{r} \frac{d}{d t}\right) i_{r q}$,

$J \frac{d \Omega}{d t}=T_{w}+T$

$\frac{d \beta}{d t}+C_{\beta} \beta=C_{\beta} u$

in which,

$$
\begin{aligned}
\widetilde{R}_{s} & =R_{s}+R_{c}, \quad \omega=G P \Omega=(1-s) \omega_{0}, \\
\tilde{L}_{s} & =L_{s}+L_{c}, \quad T=\frac{3}{2} M P G\left(i_{s q} i_{r d}-i_{s d} i_{r q}\right), \\
\gamma & =\frac{R \Omega}{V_{w}(t)}, \quad P_{e}=\omega T, \\
K_{t} & =\frac{\rho \pi R^{3}}{2}, \quad T_{\omega}=C_{t} K_{t} V_{w}^{2}(t), \\
C_{t} & =\left(-C_{1} \beta-C_{2}\right) \gamma+\left(-C_{3} \beta+C_{4}\right) .
\end{aligned}
$$

\section{State Equations of Windmill Power System}

In the dynamic equations (1), the deviations of the currents, the angular velocity and the pitch angle from their nominal values are considered to be new state variables. We have the following state equations of the windmill power system: 


$$
\begin{aligned}
& \frac{d \boldsymbol{x}}{d t}=\boldsymbol{A} \boldsymbol{x}+\widetilde{\omega} \boldsymbol{A}_{2}\left(\boldsymbol{x}+\boldsymbol{i}^{*}\right), \\
& \frac{d \widetilde{\omega}}{d t}=g_{1}(\widetilde{\omega}, t) \widetilde{\beta}+g_{2}(\boldsymbol{x}, \widetilde{\omega}, t), \\
& \frac{d \tilde{\beta}}{d t}=-C_{\beta} \widetilde{\beta}+C_{\beta} \widetilde{u}, \ldots \ldots
\end{aligned}
$$

where

$$
\begin{aligned}
& \boldsymbol{x}=\left[x_{1}, x_{2}, x_{3}, x_{4}\right]^{T}, \quad i^{*}=\left[i_{s d}^{*}, i_{s q}^{*}, i_{r d}^{*}, i_{r q}^{*}\right]^{T}, \\
& \boldsymbol{A}=\boldsymbol{A}_{1}+\widetilde{\omega}^{*} \boldsymbol{A}_{2} \\
& g_{1}(\widetilde{\omega}, t)=\widetilde{C}_{1}(t) \widetilde{w}+\widetilde{C}_{1}(t) \omega^{*}+\widetilde{C}_{3}(t), \\
& g_{2}(x, \widetilde{\omega}, t)=\boldsymbol{A}_{3} x+\left\{\widetilde{C}_{1}(t) \beta^{*}+\widetilde{C}_{2}(t)\right\} \widetilde{\omega} \\
& +\widetilde{M}\left(x_{2} x_{3}-x_{1} x_{4}\right) \\
& \boldsymbol{A}_{1}=\frac{1}{\Delta}\left[\begin{array}{cccc}
-\widetilde{R}_{s} L_{r} & \Delta \omega_{0} & R_{r} M & 0 \\
-\Delta \omega_{0} & -\widetilde{R}_{s} L_{r} & 0 & R_{r} M \\
\widetilde{R}_{s} M & 0 & -R_{r} \widetilde{L}_{s} & \Delta \omega_{0} \\
0 & \widetilde{R}_{s} M & -\Delta \omega_{0} & -R_{r} \widetilde{L}_{s}
\end{array}\right] \\
& A_{2}=\frac{1}{\Delta}\left[\begin{array}{cccc}
0 & M^{2} & 0 & L_{r} M \\
-M^{2} & 0 & -L_{r} M & 0 \\
0 & -\widetilde{L}_{s} M & 0 & -\widetilde{L}_{s} L_{r} \\
\widetilde{L}_{s} M & 0 & \widetilde{L}_{s} L_{\tau} & 0
\end{array}\right] \text {, } \\
& \boldsymbol{A}_{3}=\widetilde{M}\left[\begin{array}{llll}
-i_{r q}^{*} & i_{r d}^{*} & i_{s q}^{*} & -i_{s d}^{*}
\end{array}\right], \\
& \Delta=\widetilde{L}_{s} L_{r}-M^{2} \text {, } \\
& \widetilde{C}_{1}(t)=-\frac{C_{1} R K_{t} V_{w}(t)}{J}, \quad \widetilde{C}_{2}(t)=-\frac{C_{2} R K_{t} V_{w}(t)}{J} \\
& \widetilde{C}_{3}(t)=-\frac{C_{3} P G K_{t} V_{w}^{2}(t)}{J}, \quad \widetilde{M}=\frac{3 M P^{2} G^{2}}{2 J} .
\end{aligned}
$$

In the above equations, $x_{1}, x_{2}, x_{3}, x_{4}$ are the deviations of the stator currents and the rotor currents of the $(d-q)$ axes from their nominal values $i_{s d}^{*}, i_{s q}^{*}, i_{r d}^{*}, i_{r q}^{*} ; \widetilde{\omega}$ is the deviations of the electric angular velocity of the rotor from its nominal value $\omega^{*}=\left(1-s_{0}\right) \omega_{0} ; \widetilde{\beta}$ and $\widetilde{u}$ are deviations from their nominal values $\beta^{*}$ and $u^{*}$. The rated slip ratio is expressed by $s_{0}$. It is assumed that $\Delta \neq 0$, which is always satisfied in practical generator systems ${ }^{(6)}$.

\section{Control Law under Full-state Measurement}

Since the windmill power system has a strong nonlinearity, and it has time varying coefficients $\widetilde{C}_{1}(t), \widetilde{C}_{2}(t)$ and $\widetilde{C}_{3}(t)$ which depend on wind speed $V_{w}(t)$, it is difficult to construct a stable pitch controller. Here, we present a nonlinear Lyapunov-based stabilizing controller which regulates the pitch angle of the blade in the windmill power system, and thus regulates the state vector $\boldsymbol{x}$ and angular velocity around the rated equilibrium point.

Assume that generator inductances and resistances are exactly known, so that matrices $\boldsymbol{A}_{1}, \boldsymbol{A}_{2}, \boldsymbol{A}_{3}$ and $A$ are known. In this section we assume that the state vector $x$ is available. The output-feedback counterpart is considered in section 6 .

In order to find a Lyapunov function which assures the stability of the windmill power system, we introduce two intermediate variables of $\bar{u}$ and $z$.

Here, we define

$$
\widetilde{\beta} \triangleq \frac{1}{g_{1}(\widetilde{\omega}, t)}(\bar{u}+z),
$$

Putting (5) into (3), we obtain

$$
\frac{d \tilde{\omega}}{d t}=\bar{u}+z+g_{2}(\boldsymbol{x}, \widetilde{\omega}, t) .
$$

We shall study the stability of the windmill power system on the basis of Lyapunov's direct method. Because $\boldsymbol{A}$ is a stable matrix, by giving a positive definite matrix $Q$, we find the symmetric positive-definite matrix $P$ which satisfies Lyapunov equation $\boldsymbol{P} \boldsymbol{A}+\boldsymbol{A}^{T} \boldsymbol{P}=-\boldsymbol{Q}$.

Let us introduce the following Lyapunov function candidate $\mathrm{V}(\mathbf{t})$ :

$$
V(t) \triangleq V_{1}(t)+V_{2}(t)+V_{3}(t)
$$

in which $V_{1}(t) \triangleq \boldsymbol{x}^{T} \boldsymbol{P} \boldsymbol{x}, V_{2}(t) \triangleq \frac{1}{2} \widetilde{\omega}^{2}, V_{3}(t) \triangleq \frac{1}{2} z^{2}$.

Taking the time derivative of $(7)$, we obtain

$$
\frac{d V(t)}{d t}=\frac{d V_{1}(t)}{d t}+\frac{d V_{2}(t)}{d t}+\frac{d V_{3}(t)}{d t}
$$

Differentiating $V_{1}$ along (2) gives

$$
\begin{aligned}
\frac{d V_{1}(t)}{d t}=- & \boldsymbol{x}^{T} \boldsymbol{Q} \boldsymbol{x}+\widetilde{\omega} \boldsymbol{x}^{T}\left(\boldsymbol{P} \boldsymbol{A}_{2}+\boldsymbol{A}_{2}^{T} \boldsymbol{P}\right) \boldsymbol{x} \\
& +2 \widetilde{\omega} \boldsymbol{x}^{T} \boldsymbol{P} \boldsymbol{A}_{2} i^{*}
\end{aligned}
$$

From (6), we have

$$
\frac{d V_{2}(t)}{d t}=\widetilde{\omega}\left[\bar{u}+z+g_{2}(\boldsymbol{x}, \widetilde{\omega}, t)\right] .
$$

Define

$$
\begin{aligned}
\bar{u} \triangleq & -g_{2}(\boldsymbol{x}, \widetilde{\omega}, t)-\boldsymbol{x}^{T}\left(\boldsymbol{P} \boldsymbol{A}_{2}+\boldsymbol{A}_{2}^{T} \boldsymbol{P}\right) \boldsymbol{x} \\
& -2 \boldsymbol{x}^{T} \boldsymbol{P} \boldsymbol{A}_{2} i^{*}-\lambda_{1} \widetilde{\omega}
\end{aligned}
$$

in which $\lambda_{1}$ is an arbitrary design parameter.Putting (11) into (10), we obtain

$$
\begin{array}{r}
\frac{d V_{2}(t)}{d t}=\widetilde{\omega}\left[z-\boldsymbol{x}^{T}\left(\boldsymbol{P} \boldsymbol{A}_{2}+\boldsymbol{A}_{2}^{T} \boldsymbol{P}\right) \boldsymbol{x}\right. \\
\left.-2 \boldsymbol{x}^{T} \boldsymbol{P} \boldsymbol{A}_{2} i^{*}-\lambda_{1} \tilde{\omega}\right]
\end{array}
$$

Next,

$$
\frac{d V_{3}(t)}{d t}=z \frac{d z}{d t}
$$

From (5) and (4), it follows that 


$$
\begin{array}{r}
\frac{d z}{d t}=\tilde{\beta} \frac{d g_{1}(\widetilde{\omega}, t)}{d t}-C_{\beta} g_{1}(\tilde{\omega}, t) \tilde{\beta} \\
+C_{\beta} g_{1}(\widetilde{\omega}, t) \tilde{u}-\frac{d \bar{u}}{d t} .
\end{array}
$$

Let the control law be

$$
\begin{aligned}
& \tilde{u} \triangleq \frac{-1}{C_{\beta} g_{1}(\widetilde{\omega}, t)} \times \\
& {\left[\widetilde{\beta} \frac{d g_{1}(\widetilde{\omega}, t)}{d t}-C_{\beta} g_{1}(\widetilde{\omega}, t) \tilde{\beta}-\frac{d \bar{u}}{d t}+\lambda_{2} z\right],}
\end{aligned}
$$

where $\lambda_{2}$ is an arbitrary design parameter.

Substituting (15) in (14) gives

$$
\frac{d z}{d t}=-\lambda_{2} z
$$

Obviously, $\mathrm{z}$ is exponentially stable.

From (13) and (16),we have

$$
\frac{d V_{3}(t)}{d t}=-\lambda_{2} z^{2}
$$

By putting (9),(10) and (17) into (8), we finally get

$$
\begin{aligned}
\frac{d V(t)}{d t} & =-x^{T} Q x+\widetilde{\omega} z-\lambda_{1} \widetilde{\omega}^{2}-2 \lambda_{2} z^{2} \\
& \leq-\mu_{1} V_{1}+\mu_{2} \sqrt{V_{2}}-\lambda_{1} V_{2}-2 \lambda_{2} V_{3} \\
& \leq-\delta_{1} V(t)+\delta_{2} e^{-2 \lambda_{2} t}, \ldots \ldots \ldots \ldots
\end{aligned}
$$

in which

$$
\begin{array}{ll}
\mu_{1} \triangleq \frac{\lambda_{\min }(\boldsymbol{Q})}{\lambda_{\max }(\boldsymbol{P})}, & \mu_{2} \triangleq z(0) e^{-\lambda_{2} t} \\
\delta_{1} \triangleq \min \left(\mu_{1}, \frac{1}{2} \lambda_{1}, 2 \lambda_{2}\right), & \delta_{2} \triangleq \frac{1}{2 \lambda_{1}} z^{2}(0)
\end{array}
$$

where the $\lambda_{\min }(\boldsymbol{Q})$ and $\lambda_{\max }(\boldsymbol{P})$ denote the smallest and largest eigenvalue of $\boldsymbol{Q}$ and $\boldsymbol{P}$ respectively.

The solution of differential inequality (18) is given by

$$
\begin{array}{ll}
V(t) \leq \delta_{2} t e^{-\delta_{1} t}+V(0) e^{-\delta_{2} t} & \delta_{1}=2 \lambda_{2} . \\
V(t) \leq \frac{\delta_{2}}{\delta_{1}-2 \lambda_{2}} e^{-2 \lambda_{2} t}+V(0) e^{-\delta_{1} t} & \delta_{1} \neq 2 \lambda_{2} .
\end{array}
$$

Since $\lim _{t \rightarrow \infty} V(t) \rightarrow \cdot 0$, we conclude that $x, \tilde{\omega}, z$ are globally stable. As $x$ and $\tilde{\boldsymbol{\omega}}$ are the deviations from their nominal values and $z$ is exponentially globally stable, $x, \widetilde{w}$ and $z$ converge on zero. Consequently, by the definition of $\bar{u}$ in (11), we know that $\bar{u}$ converges on zero; and from (5), $\tilde{\beta}$ converges on zero. Hence the windmill power system is globally stable under the control law of (15).

\section{Observer of Windmill Power System}

In implementing the controller, it is difficult to physically measure the rotor currents, that is, the state variables $\left(x_{3}, x_{4}\right)$, so a control design based on estimation of the state variables $\left(x_{3}, x_{4}\right)$ is required. We assume that the stator currents, the electric angular velocity of the induction generator and the pitch angle are measurable, so that the observer which estimates the rotor currents of the generator is to be constructed. We shall prove that the observer of the generator subsystem (2) is given by

$\frac{d \widehat{x}}{d t}=A \widehat{x}+\widetilde{\omega} A_{2}\left(\widehat{x}+i^{*}\right)-F(y-C \widehat{x})-\widetilde{\omega} \boldsymbol{K}(\boldsymbol{y}-C \widehat{x})$,

where $\widehat{x}$ denotes estimates of $\boldsymbol{x}, \boldsymbol{C}=\left[\begin{array}{llll}1 & 0 & 0 & 0 \\ 0 & 1 & 0 & 0\end{array}\right]$ and the observer gains are determined by

$\boldsymbol{F}=\frac{1}{\Delta}\left[\begin{array}{ll}f_{11} & f_{12} \\ f_{21} & f_{22} \\ f_{31} & f_{32} \\ f_{41} & f_{42}\end{array}\right], \quad \boldsymbol{K}=\frac{1}{\Delta}\left[\begin{array}{ll}k_{11} & k_{12} \\ k_{21} & k_{22} \\ k_{31} & k_{32} \\ k_{41} & k_{42}\end{array}\right]$

in which $f_{i j}$ and $k_{i j}$ are given by

$k_{11}=k_{12}=k_{21}=k_{22}=k_{31}=k_{42}=0$,

$k_{32}=\left(\widetilde{L}_{s}+L_{r}\right) M, \quad k_{41}=\left(-\widetilde{L}_{s}-L_{r}\right) M$

$f_{11}=f_{12}=f_{21}=f_{22}=0$,

$f_{32}=\omega^{*}\left(\widetilde{L}_{s}+L_{r}\right) M, \quad f_{41}=\omega^{*}\left(-\widetilde{L}_{s}-L_{r}\right) M$

$f_{31}=f_{42}=-\left(\widetilde{R}_{s}+R_{r}\right) M$.

Proof: Let

$$
\boldsymbol{e}=\boldsymbol{x}-\widehat{\boldsymbol{x}},
$$

therefore, the error dynamics between the designed observer (21) and the induction generator model (2) is derived:

$$
\frac{d e}{d t}=(\boldsymbol{A}+\boldsymbol{F C}) \boldsymbol{e}+\widetilde{\omega}\left(\boldsymbol{A}_{2}+\boldsymbol{K} \boldsymbol{C}\right) \boldsymbol{e} .
$$

We define Lyapunov function $V_{e} \triangleq \frac{1}{2} \|\left.\boldsymbol{e}\right|^{2}$. Then, from (25) we have

$$
\begin{aligned}
\frac{d V_{e}}{d t}= & e^{T}\left[(\boldsymbol{A}+\boldsymbol{F C})+(\boldsymbol{A}+\boldsymbol{F} \boldsymbol{C})^{T}\right] e \\
& +\widetilde{\omega} \boldsymbol{e}^{T}\left[\left(\boldsymbol{A}_{2}+\boldsymbol{K} \boldsymbol{C}\right)+\left(\boldsymbol{A}_{2}+\boldsymbol{K} \boldsymbol{C}\right)^{T}\right] \boldsymbol{e} .
\end{aligned}
$$

Since

$\boldsymbol{A}_{2}+\boldsymbol{K} \boldsymbol{C}=\frac{1}{\Delta}\left[\begin{array}{cccc}0 & M^{2} & 0 & L_{r} M \\ -M^{2} & 0 & -L_{r} M & 0 \\ 0 & L_{r} M & 0 & -\widetilde{L}_{s} L_{r} \\ -L_{r} M & 0 & \widetilde{L}_{s} L_{r} & 0\end{array}\right]$,

$\boldsymbol{A}_{2}+\boldsymbol{K} \boldsymbol{C}+\left(\boldsymbol{A}_{2}+\boldsymbol{K} \boldsymbol{C}\right)^{T}=\boldsymbol{O}$ is obtained; and from

$\boldsymbol{A}+\boldsymbol{F C}=\frac{1}{\Delta} \times$

$\left[\begin{array}{cccc}-\widetilde{R}_{s} L_{r} & \Delta \omega_{0}+\omega^{*} M^{2} & R_{r} M & \omega^{*} L_{r} M \\ -\Delta \omega_{0}-\omega^{*} M^{2} & -\widetilde{R}_{s} L_{r} & -\omega^{*} L_{r} M & R_{r} M \\ R_{r} M & \omega^{*} L_{r} M & -R_{r} \widetilde{L}_{s} & \Delta \omega_{0}-\omega^{*} \widetilde{L}_{s} L_{r} \\ -\omega^{*} L_{r} M & -R_{r} M & \omega^{*} \widetilde{L}_{s} L_{r}-\Delta \omega_{0} & -R_{r} \widetilde{L}_{s}\end{array}\right]$

we have

$$
\begin{aligned}
& (\boldsymbol{A}+\boldsymbol{F} \boldsymbol{C})+(\boldsymbol{A}+\boldsymbol{F} \boldsymbol{C})^{T}= \\
& \frac{-2}{\Delta} \operatorname{diag}\left[\widetilde{R}_{s} L_{r}, \widetilde{R}_{s} L_{r}, R_{r} \widetilde{L}_{s}, R_{r} \widetilde{L}_{s}\right]<\boldsymbol{O} .
\end{aligned}
$$

Therefore 


$$
\frac{d V_{e}}{d t} \leq-\frac{2}{\triangle} \delta_{e}\|e\|^{2}
$$

where

$$
\delta_{e}=\min \left\{\widetilde{\mathrm{R}}_{\mathrm{s}} \mathrm{L}_{\mathrm{r}}, \widetilde{\mathrm{R}}_{\mathrm{s}} \mathrm{L}_{\mathrm{r}}, \mathrm{R}_{\mathrm{r}} \widetilde{\mathrm{L}}_{\mathrm{s}}, \mathrm{R}_{\mathrm{r}} \widetilde{\mathrm{L}}_{\mathrm{s}}\right\} .
$$

Hence, $\lim _{t \rightarrow \infty} V_{e}(t) \rightarrow 0$. It is therefore proved that (25) is globally asymptotically stable.

\section{Observer-based Controller of Windmill Pow- er System}

The controller design under full-state measurement and the rotor current observer design were studied in section 4 and section 5 respectively. Here we show that the controller can be combined with the observer while retaining the global stability properties.

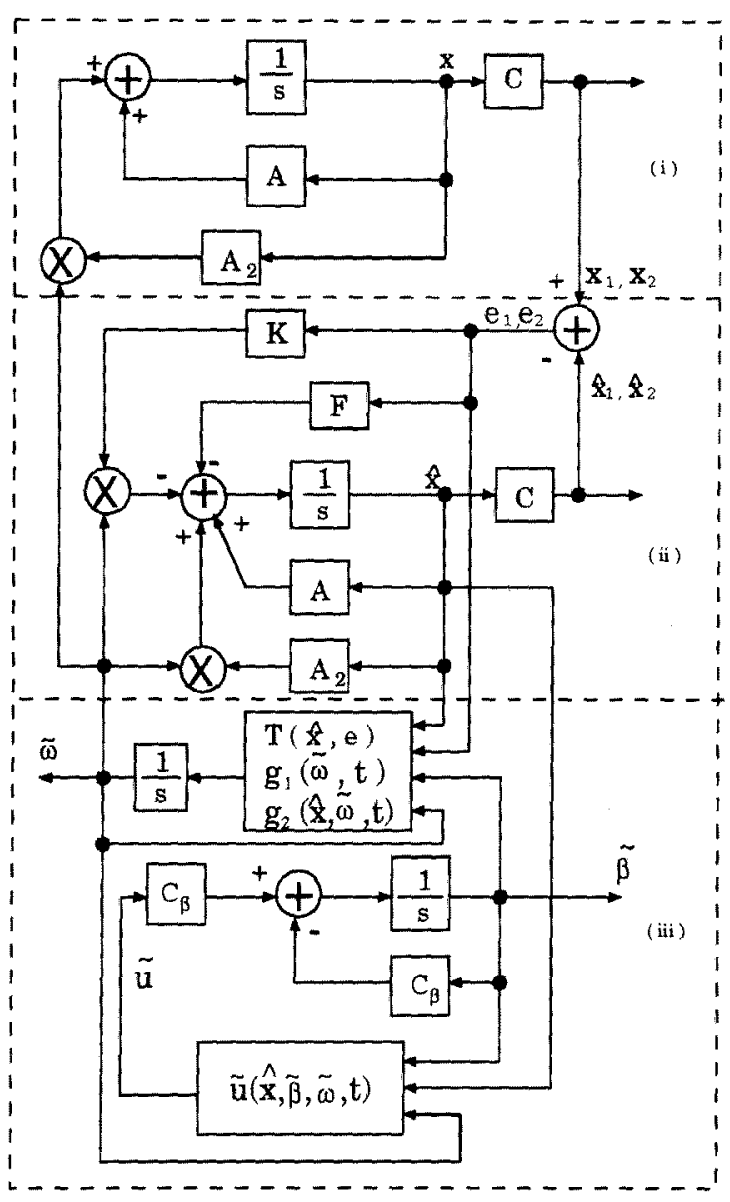

Fig. 3. Block diagram of the observer-based windmill power system.

The block diagram of the observer-based windmill power system is shown in Fig. 3. It consists of

(i) the induction generator subsystem;

(ii) the rotor currents observer;

(iii) the windmill and hydraulic system.

Considering the definition (24) and the observer (21) with the windmill power system (2), (3) and (4), we have the following observer-based windmill power system:

$$
\begin{aligned}
& \frac{d \widehat{x}}{d t}=A \widehat{x}+\widetilde{\omega} \boldsymbol{A}_{2}\left(\widehat{\boldsymbol{x}}+i^{*}\right)-\boldsymbol{F C} \boldsymbol{e}-\widetilde{\omega} \boldsymbol{K} \boldsymbol{C e}, \\
& \frac{d \widetilde{\omega}}{d t}=g_{1}(\widetilde{\omega}, t) \tilde{\beta}+g_{2}(\widehat{\boldsymbol{x}}, \widetilde{\omega}, t)+T(\widehat{\boldsymbol{x}}, \boldsymbol{e}) \ldots \ldots \\
& \frac{d \widetilde{\beta}}{d t}=-C_{\beta} \widetilde{\beta}+C_{\beta} \tilde{u}_{e}, \ldots \ldots \ldots \ldots \ldots \ldots \ldots
\end{aligned}
$$

in which

$$
\begin{array}{ll}
\widehat{\boldsymbol{x}}=\left(\widehat{x}_{1}, \widehat{x}_{2}, \widehat{x}_{3}, \widehat{x}_{4}\right)^{T}, & \boldsymbol{e}=\left(\epsilon_{1}, e_{2}, 0,0\right)^{T} . \\
T(\widehat{\boldsymbol{x}}, \boldsymbol{e})=\boldsymbol{A}_{3} \boldsymbol{e}+\widetilde{M} \boldsymbol{e} \widehat{x}, & \boldsymbol{e}^{\prime}=\left(\mathbf{0}, 0, e_{2},-e_{1}\right) .
\end{array}
$$

Note that this structure differs from that of (2), (3) and (4) by the fact that we use estimated values of the state variables. In order to study the stability of the observerbased windmill power system, we recall (5) and (7), and follow the same reasoning as in section 4 .

Define

$$
\widetilde{\beta} \triangleq \frac{1}{g_{1}(\widetilde{\omega}, t)}\left(\bar{u}_{e}+z\right)
$$

then

$$
\begin{aligned}
& \bar{u}_{e} \triangleq-g_{2}(\widehat{\boldsymbol{x}}, \tilde{\omega}, t)-\widehat{\boldsymbol{x}}^{T}\left(\boldsymbol{P} \boldsymbol{A}_{2}+\boldsymbol{A}_{2}^{T} \boldsymbol{P}\right) \widehat{\boldsymbol{x}} \\
& -2 \widehat{\boldsymbol{x}}^{T} \boldsymbol{P} \boldsymbol{A}_{2} i^{*}-\lambda_{1} \tilde{\omega}, \\
& \tilde{u}_{e} \triangleq \frac{-1}{C_{\beta} g_{1}(\widetilde{\omega}, t)} \times \\
& {\left[\tilde{\beta} \frac{d g_{1}(\widetilde{\omega}, t)}{d t}-C_{\beta} g_{1}(\widetilde{\omega}, t) \widetilde{\beta}-\frac{d \bar{u}_{e}}{d t}+\lambda_{2} z\right] .}
\end{aligned}
$$

Considering the Lyapunov function candidate

$$
V_{e} \triangleq V_{e 1}(t)+V_{\mathrm{e} 2}(t)+V_{e 3}(t)
$$

where $V_{e 1}(t) \triangleq \widehat{\boldsymbol{x}}^{T} \boldsymbol{P} \widehat{\boldsymbol{x}}, V_{\mathrm{e} 2}(t) \triangleq \frac{1}{2} \widetilde{\omega}^{2}, V_{\mathrm{e} 3}(t) \triangleq$ $\frac{1}{2} z^{2}$. Taking the derivative of (33) with respect to time and considering the definitions (31) and (32), we get

$$
\begin{array}{r}
\frac{d V_{e}(t)}{d t}=-\widehat{\boldsymbol{x}}^{T} \boldsymbol{Q} \tilde{\boldsymbol{x}}-2 \widehat{\boldsymbol{x}}^{T} \boldsymbol{P F C e}-2 \widetilde{\omega} \widehat{\boldsymbol{x}}^{T} \boldsymbol{P} \boldsymbol{K} \boldsymbol{C e} \\
+\widetilde{\omega}\left(z-\lambda_{1} \tilde{\omega}\right)-\lambda_{2} z^{2}+\widetilde{\omega}\left(\boldsymbol{A}_{3} e+\widetilde{M} e^{\prime} \widehat{\boldsymbol{x}}\right) \\
\leq-\mu_{1} V_{e 1}+\mu_{2} \sqrt{V_{e 2}}+\mu_{3} \sqrt{V_{e 1}} \sqrt{V_{e 2}}+\mu_{4} \sqrt{V_{e 1}} \\
-\lambda_{1} V_{e 2}-2 \lambda_{2} V_{e 3}+\mu_{5} \sqrt{V_{e 1}} \sqrt{V_{e 2}}+\mu_{6} \sqrt{V_{e 2}}
\end{array}
$$

in which

$$
\begin{aligned}
& \mu_{1} \triangleq \frac{\lambda_{\min }(\boldsymbol{Q})}{\lambda_{\max }(\boldsymbol{P})} \quad, \mu_{2} \triangleq z(0) e^{-\lambda_{2} t}, \\
& \mu_{3} \triangleq \sup _{t \geq 0}\|-2 \boldsymbol{P} \boldsymbol{K C e}\|, \mu_{4} \triangleq \sup _{t \geq 0}\|-2 \boldsymbol{P F C e}\|, \\
& \mu_{5} \triangleq \sup _{t \geq 0}\|-2 \widetilde{M} \boldsymbol{e}\| \quad, \mu_{6} \triangleq \sup _{t \geq 0}\left\|-2 \boldsymbol{A}_{3} \boldsymbol{e}\right\| .
\end{aligned}
$$

Following the same reasoning as in section 4 , we get 


$$
\begin{array}{r}
\frac{d V_{\mathrm{e}}(t)}{d t} \leq-\frac{1}{4} \mu_{1} V_{\mathrm{e} 1}-\frac{1}{2} \lambda_{1}^{\prime} V_{\mathrm{e} 2}- \\
2 \lambda_{2} V_{\mathrm{e} 3}+\frac{\mu_{2}^{2}}{\mu_{1}}+\frac{\left(\mu_{4}+\mu_{6}\right)^{2}}{2 \lambda^{\prime}}
\end{array}
$$

where

$$
\lambda_{1}^{\prime} \triangleq \lambda_{1}-\frac{\left(\mu_{3}+\mu_{5}\right)^{2}}{2 \mu_{1}} .
$$

Since $\lambda_{1}$ is an arbitrary design parameter, choosing $\lambda_{1}$ sufficiently large yields $\lambda_{1}^{\prime} \geq 0$. Finally we rewrite (35) in a more compact form as

$$
\frac{d V_{e}(t)}{d t} \leq-\delta_{1}^{\prime} V_{e}(t)+\delta_{2}^{\prime} e^{-k t}
$$

in which

$$
\begin{aligned}
& \delta_{1}^{\prime} \triangleq \min \left\{\frac{1}{4} \mu_{1}, \frac{1}{2} \lambda_{1}^{\prime}, 2 \lambda_{2}\right\}, \\
& \delta_{2}^{\prime} e^{-k t} \geq \frac{\mu_{2}^{2}}{\mu_{1}}+\frac{\left(\mu_{4}+\mu_{6}\right)^{2}}{2 \lambda_{1}^{\prime}} .
\end{aligned}
$$

As $\mu_{2}, \mu_{4}$ and $\mu_{6}$ are exponentially stable that $\lim _{t \rightarrow \infty} \delta_{2}^{\prime} e^{-k t} \rightarrow 0$. The solution of differential inequality (36) is given by

$$
\begin{array}{ll}
V_{e}(t) \leq \delta_{2}^{\prime} t e^{-k t}+V_{e}(0) e^{-\delta_{1}^{\prime} t} & \delta_{1}^{\prime}=k \\
V_{e}(t) \leq \frac{\delta_{2}^{\prime}}{\delta_{1}^{\prime}-k} e^{-k t}+V_{e}(0) e^{-\delta_{1}^{\prime} t} & \delta_{1}^{\prime} \neq k
\end{array}
$$

Since $\lim _{t \rightarrow \infty} V_{e}(t) \rightarrow 0$, we conclude that the windmil1 power system combined with the observer is globally stable.

\section{Simulation results}

In order to verify the effectiveness of the proposed controller and observer which regulate the windmil1 power system to keep the rated value of speed and output in the range of high wind speed, a simulation was carried out. Validation of the scheme is tested by the windmill induction generator system which is connected to infinite bus as shown in Fig.1.

The system coefficients and rated value of the windmill induction generator system, which consists of the windmill induction generator, the hydraulic system for

Table 1. System coefficients and rated values.

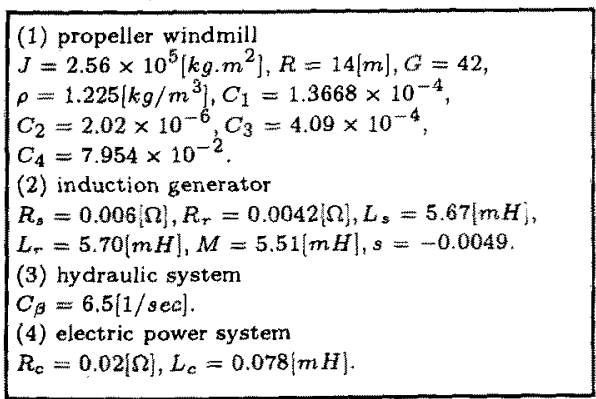

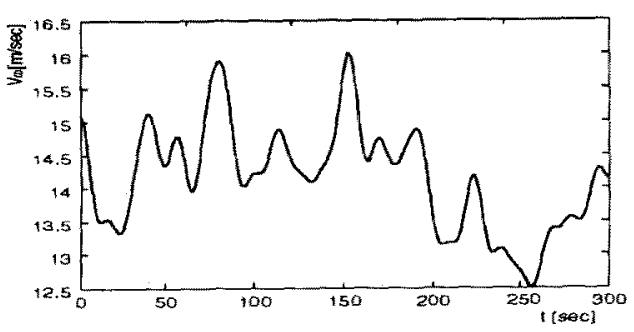

Fig. 4. The actual wind speed.

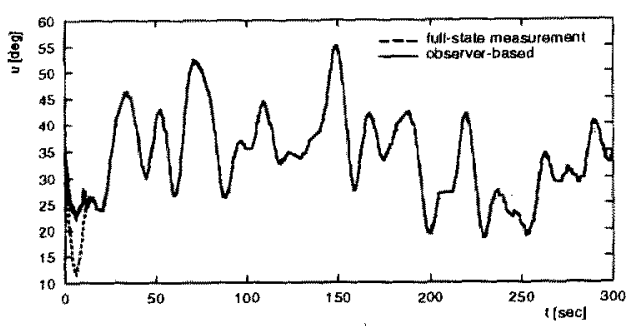

Fig. 5. The control u.

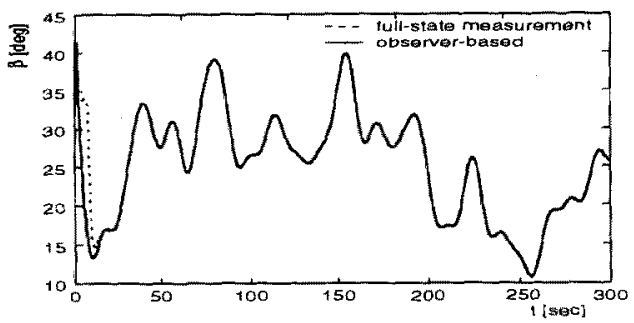

Fig. 6. The pitch angle of blade.

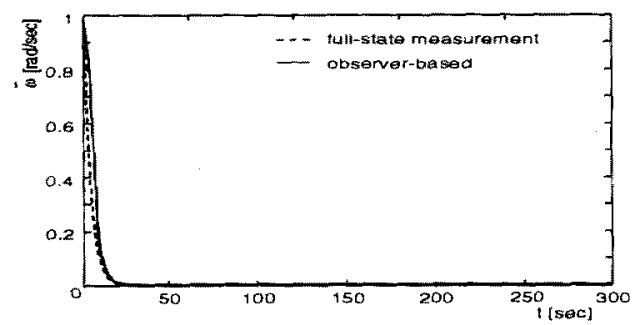

Fig. 7. The electric angular velocity of generator.

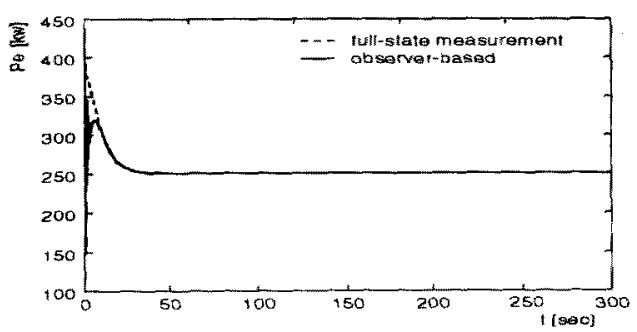

Fig. 8. The power output of the windmill power system. 
driving pitch angle and the electrical power system, are shown in table 1. They are supplied by Mitsubishi Heavy Industries, LTD. in Japan.

In the simulations, the test wind pattern was made from the actual wind speed data measured at Miyako Island, Okinawa, Japan in December 1994, which is shown in Fig.4. The wind speed range limits, the voltages of the windmill system and the control design parameters are shown in table 2 .

Table 2. Control system design parameters

(1) speed range limits of wind
$V_{i n}(t)=5.5[\mathrm{~m} / \mathrm{s}], V_{\text {out }}(t)=25[\mathrm{~m} / \mathrm{s}]$
$V^{*}(t)=12.4[\mathrm{~m} / \mathrm{s}]$
$(2)$ voltages of the windmill power system
$V_{s d}=480[V], V_{s Q}=480[V], V_{\text {bus }}=6600[\mathrm{~V}]$
$(3)$ design parameters
$Q=$ diag $[2.579,2.579,2.579,2.579]$
$\lambda_{1}=16.8, \lambda_{2}=4.5$

Fig. 5 shows the behavior of control $u$ which brings about the change of pitch angle of blade with the change of wind speed so as to get stable electric angular velocity and power output. Fig. 6 and 7 show the behavior of pitch angle of blade and electric angular velocity of generator in the windmill power system for both full-state measurement and observer-based cases. Fig. 8 shows the power output of the windmill power system. The character of deviation of the angular velocity and the power output show that the deviation of the angular velocity and the change of wind speed is restrained sufficiently and they converge on the rated values quickly by using the controller and observer proposed in this paper.

As demonstrated above, when the controller and observer proposed in the paper is used, though the wind speed which is in the range of high wind speed changes, the deviation of the angular velocity and the power output of the windmill induction generator is improved. What is more, the difficulty of physically measuring the rotor currents is overcome by using the observer designed in the paper. The simulation results show that transient stability of the windmill power system is effectively obtained by using the proposed controller and observer.

\section{Conclusions}

This paper has used Lyapunov's direct method to design nonlinear controller and observer for the windmil1 power system. The proposed controller and observer not only enable global asymptotical stabilization of the plants, but also effectively keep the generated power of wind turbine generators at the rated value under the varying wind speed. They were tested through an example for both full-state measurement system and observer-based extended system. The results show that transient stability of the windmill power system is effectively obtained by using the proposed controller and observer. 11)

\section{References}

(1) O. Kanna, S. Hanba, and K. Yamashita: "A method of damping transient rush voltage fluctuation of power systems due to a wind driven generator via nonlinear state feedback control" , T. IEE Japan B, Vol. 117-B, No. 4, pp. 572-577 (1997)

(2) O. Kanna, S. Hanba, S. Asato and K. Yamashita: "A method of Stabilization of a Wind Generator Power Using Backstepping Algorithm" , T. IEE Japan B, Vol. 117-B, No. 12, pp. 1513-1519 (1997)

(3) S. Hara and K. Furuta: "Minimal order state observers for bilinear System", Internat. J. Control, Vol. 24, No. 5, pp. $705-718(1976)$

(4) Y. Funahashi: "Stable state estimator for bilinear systems", Internat. J. Control, Vol. 29, No. 2, pp. 181-188 (1979)

(5) H. J. Shieh, K. K. Shyu and F. J. Lin: "Adaptive estimation of rotor time constant for indirect, field-oriented induction motor drive", IEE Proc. Electr. Power Appl., Vol. 145, (2), pp. $111-118,(1998)$.

(6) Y. Y. Wang and D. J. H.ll: "Robust nonlinear coordinated control of Power system", Automatica, Vol. 32, No. 4, pp. 611-618, (1996).

(7) C. C. De Wit and S. I. Seleme JI: "Robust torque control design for induction motors: the minimum energy approach", Automatica, Vol. 33 , No. 1, pp. 63.79, (1997).

\section{Appendix}

List of symbols:

$R_{s}:$ stator resistance $[\Omega]$.

$R_{r}$ : rotor resistance $[\Omega]$.

$L_{s}$ : stator inductance $[\mathrm{H}]$.

$L_{r}$ : rator inductance $[\mathrm{H}]$.

$\mathrm{M}$ : mutual inductance between rotor coil and stator $\operatorname{coil}[\mathrm{H}]$

$R_{c}$ : power-transmission line resistance calculated to generator side $[\Omega]$

$L_{c}$ : power-transmission line inductance calculated to generator side $[\Omega]$.

$\omega$ : electric angular velocity $[\mathrm{rad} / \mathrm{sec}]$.

$\omega_{0}$ : synchronous angular velocity $[\mathrm{rad} / \mathrm{sec}]$.

$\Omega$ : mechanical angular velocity [ $\mathrm{rad} / \mathrm{sec}]$.

s: slip of angular velocity.

$\mathrm{J}$ : inertia moment of windmill $\left[\mathrm{kg} \cdot \mathrm{m}^{2}\right]$.

$T_{w}$ : torque of windmill $[\mathrm{J}]$.

$\mathrm{T}$ : load torque of induction generator $[\mathrm{J}]$.

$P_{e}$ : output of induction generator [w].

$P$ : pole pairs of induction generator.

G: gear ratio of gearbox.

$\beta$ : pitch angle of blade of windmill [deg].

$C_{t}$ : coefficient of torque

$C_{1}, C_{2}, C_{3}, C_{4}$ : coefficient of windmill.

$C_{\beta}$ : coefficient of hydraulic system.

$\gamma$ : angular speed rate of windmill.

$\rho$ : density of air.

$\mathrm{R}$ : radius of windmill [m].

$\mathrm{u}$ : control input.

$V_{w}(t)$ : speed of wind [m/sec].

$V_{\text {in }}(t)$ : cut-in speed of wind.

$V_{\text {out }}(t)$ : cut-out speed of wind.

$V^{*}(t):$ rated speed of wind

$V_{s d}, V_{s q}$ : d axis and $\mathrm{q}$ axis voltages of generator which is calculated from the infinite bus [V].

$V_{b u s}$ : voltage in the side of infinite bus [V].

$i_{s d}, i_{s q}: \mathrm{d}$ axis and $\mathrm{q}$ axis currents of stator [A]. 
$i_{r d}, i_{r q}: \mathrm{d}$ axis and $\mathrm{q}$ axis currents of rotor $[\mathrm{A}]$.

Youjiang Long (Student Member) was born in China,in

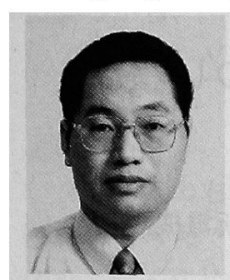
1962. He received the Bachelor's degree in electrical engineering, in 1982 , and the $M$. S. degree in control engineering from Harbin Institute of Technology, China, in 1988. Currently, he is studying in the department of electrical and electronic engineering, University of the Ryukyus, Okinawa, Japan, for $\mathrm{Ph}$. D. degree. His main research interests include robust control, adaptive control, microprocessorbased systems, and computer algorithms.

Shigeru Hanba (Non-member) completed his doctoral

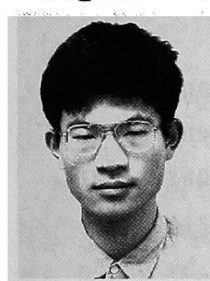
course at the School of Mathematical and Physical Sciences of the Graduate University for Advanced Studies in 1996. He has been an assistant in the Faculty of Engineering of the University of the Ryukyus since that year. He is engaged maily in research on nonlinear control, adaptive control, and sliding-mode control. He is a member of the Society of Instrument and Control Engineers of Japan. He has

\section{a $\mathrm{Ph} . \mathrm{D}$ degree.}

Hayao Miyagi (Member) completed his doctoral course at the University of Osaka Prefecture in 1977, and became an assistant in the Faculty of Engineering of University of the Ryukyus in the same year. After serving as an associate professor from 1982, he is currently a professor. $\mathrm{He}$ is engaged mainly in reseach on system stability and fuzzy control theory. He is a member of the Society of Fuzzy Theory and System Engineers of Japan, Information Processing Engineers of Japan, and the IEEE. He has a Doctor of Engineering degree.

Katsumi Yamashita (Member) completed his doctoral

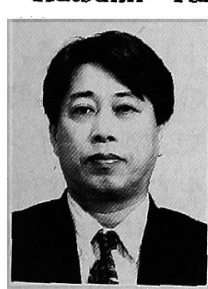
course at the University of Osaka Prefecture. He became an assistant in the Faculty of Engineering of University of the Ryukyus in 1982, and after serving as an associate professor, he is currently a professor. He is engaged mainly in reseach on adaptive control and signal processing. He is a member of the Society of Instrument and Control Engineers of Japan, the Institute of Electronics, Information and Communication Engineers of Japan, and the IEEE. He has a Doctor of Engineering degree. 